\title{
Functional defects in transfer RNAs lead to the accumulation of ribosomal RNA precursors
}

\author{
JACOBA G. SLAGTER-JÄGER, ${ }^{1,3}$ LEOPOLD PUZIS, ${ }^{2}$ NANCY S. GUTGSELL, ${ }^{2}$ MARLENE BELFORT, ${ }^{1}$ and \\ CHAITANYA JAIN ${ }^{2}$ \\ ${ }^{1}$ Wadsworth Center, New York State Department of Health, Albany, New York 12208, USA \\ ${ }^{2}$ Department of Biochemistry and Molecular Biology, University of Miami Miller School of Medicine, Miami, Florida 33136, USA
}

\begin{abstract}
Normal expression and function of transfer RNA (tRNA) are of paramount importance for translation. In this study, we show that tRNA defects are also associated with increased levels of immature ribosomal RNA (rRNA). This association was first shown in detail for a mutant strain that underproduces tRNA ${ }^{\text {Arg2 }}$ in which unprocessed 165 and 235 rRNA levels were increased several-fold. Ribosome profiles indicated that unprocessed 23S rRNA in the mutant strain accumulates in ribosomal fractions that sediment with altered mobility. Underproduction of tRNA ${ }^{\text {Arg2 }}$ also resulted in growth defects under standard laboratory growth conditions. Interestingly, the growth and rRNA processing defects were attenuated when cells were grown in minimal medium or at low temperatures, indicating that the requirement for TRNA $^{A r g 2}$ may be reduced under conditions of slower growth. Other tRNA defects were also studied, including a defect in RNase $P$, an enzyme involved in tRNA processing; a mutation in TRNA ${ }^{\text {Trp }}$ that results in its degradation at elevated temperatures; and the titration of the tRNA that recognizes rare AGA codons. In all cases, the levels of unprocessed 165 and $23 S$ rRNA were enhanced. Thus, a range of tRNA defects can indirectly influence translation via effects on the biogenesis of the translation apparatus.
\end{abstract}

Keywords: RNA processing; tRNA; rRNA; translation; Rep helicase

\section{INTRODUCTION}

The maturation of ribosomes in prokaryotes is a highly coordinated process that entails the stepwise assembly of ribosomal proteins on rRNAs. During this process, rRNAs undergo a series of maturation steps, which are completed only when the newly synthesized ribosome becomes translationally competent (Srivastava and Schlessinger 1988, 1990). Defects in rRNA processing can be symptomatic of functional defects in ribosomal proteins, processing ribonucleases (RNases), or other factors that play a role in ribosome maturation. Even in a well-studied model organism such as Escherichia coli, the list of factors that influence rRNA processing and ribosome biogenesis is substantially incomplete (Williamson 2003). Therefore, there is considerable interest in identifying additional factors that participate in these processes.

\footnotetext{
${ }^{3}$ Present address: Duke Center for Translational Research, Department of Surgery, Duke University Medical Center, Durham, NC 27710, USA.

Reprint requests to: Chaitanya Jain, Department of Biochemistry and Molecular Biology, University of Miami Miller School of Medicine, Miami, FL 33136, USA; e-mail: cjain@med.miami.edu; fax: (305) 243-3955.

Article published online ahead of print. Article and publication date are at http://www.rnajournal.org/cgi/doi/10.1261/rna.319407.
}

During a screen of rRNA processing in a panel of E. coli mutant strains, we found that one mutant displayed elevated levels of unprocessed rRNAs. Further analysis of this strain indicated that the processing defects were due to a chromosomal deletion that reduces expression of tRNA ${ }^{\text {Arg2 }}$. Here, we present an analysis of the rRNA processing defects that arise due to reduced tRNA ${ }^{\mathrm{Arg} 2}$ expression, and show that other tRNA defects also impair rRNA processing. These results reveal a previously undescribed connection between tRNA function and rRNA processing that has broad implications for the role of tRNAs in the biogenesis of the translational machinery.

\section{RESULTS}

Characterization of a strain lacking three tRNA genes

In a screen to identify factors that affect rRNA processing, derivatives of the $E$. coli strain HMS174(DE3) containing disruptions in genes related to the $\mathrm{DExD} / \mathrm{H}$-box family (Perutka et al. 2004) were analyzed by Northern blot analysis to determine whether any of these strains exhibit changes in rRNA processing. DExD/H-box proteins are a ubiquitous class of factors that have been shown to play important 
roles in nucleic acid metabolism and can function as DNA or RNA helicases (Tanner and Linder 2001; Rocak and Linder 2004). Four probes that hybridize to sequences flanking the $5^{\prime}$ and $3^{\prime}$ ends of mature $16 \mathrm{~S}$ and $23 \mathrm{~S}$ rRNAs were used (Fig. 1A, probes \#1-\#4). All four probes hybridized more strongly with RNA derived from a strain containing a disruption in the rep gene, which encodes the DNA helicase Rep, compared with RNA from a wild-type strain, suggesting that higher levels of unprocessed rRNAs accumulate in the disruption strain (Fig. 1B).

To define this phenomenon further and to map the 5 ' ends of the unprocessed rRNAs, we performed primer extension analysis. Probes \#5 and \#6, which anneal near the 5 ' ends of $16 \mathrm{~S}$ and $23 \mathrm{~S}$ rRNAs (Fig. 1A), were expected to give reverse-transcription products of 35 and 55 nucleotides (nt), respectively, with the corresponding mature rRNAs. When primer extension was conducted using the $16 \mathrm{~S}$-specific oligonucleotide \#5, in addition to the mature RNA, precursors containing extra 5 ' sequences were also observed (Fig. 1C). The precursor containing an additional $115 \mathrm{nt}$ at the $5^{\prime}$ end corresponds to a product generated by the cleavage of rRNA by the double-strand-specific ribonuclease RNase III (data not shown; King et al. 1986). Importantly, this precursor was present at higher levels in the mutant strain, indicating a processing defect. Quantitation of the signals indicated that the ratio of this precursor to the mature 16S rRNA increased from 0.06 in the wild-type strain to 0.18 in the mutant strain.

Similarly, for $23 \mathrm{~S}$ rRNA, apart from the product that corresponds to the mature $23 \mathrm{~S}$ rRNA $5^{\prime}$ end, a precursor that contains an additional $7 \mathrm{nt}$ at the $5^{\prime}$ end was found to accumulate in the mutant strain. This precursor is also generated by RNase III cleavage of ribosomal operon RNA (King et al. 1984). The ratio of this product to the mature RNA increased from 0.08 in the wild-type strain to 0.20 in the mutant strain. The multiple bands observed for the mature $16 \mathrm{~S}$ and $23 \mathrm{~S}$ rRNAs are not currently understood. Nevertheless, these analyses confirmed that the levels of unprocessed rRNA were increased for both rRNAs in the mutant strain. Based on this observed defect, we refer to the mutant strain as HMSdrp (for defective rRNA processing).

Further genetic analysis of HMSdrp, however, indicated that the rRNA processing defect was unlikely to be due to the rep disruption. First, the rRNA processing defect could not be complemented by plasmids carrying a wild-type rep allele (data not shown). Furthermore, when a rep::kan deletion mutation was transduced into HMS174(DE3) and rRNA processing was evaluated, increased levels of unprocessed rRNAs were not found (data not shown). These observations suggested that the rRNA processing defects observed in HMSdrp are due to a secondary mutation.

To gain further insight into the basis of rRNA processing defects in HMSdrp, we performed microarray analysis on RNA derived from HMSdrp, HMS174(DE3), and HMSlacZ, a derivative containing a disruption in the lac $Z$
A

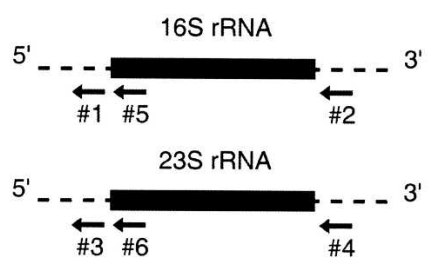

B

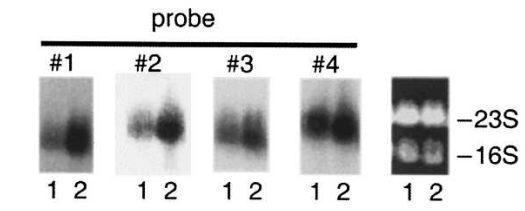

C

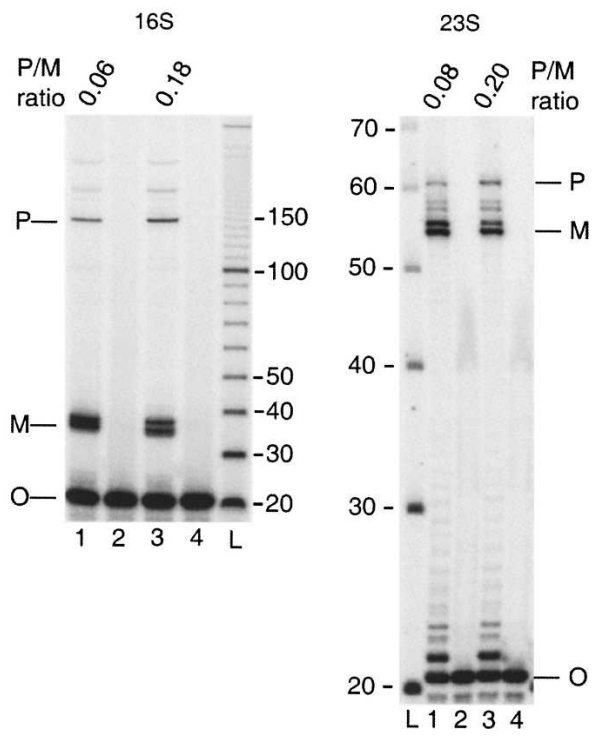

FIGURE 1. Ribosomal RNA processing defects in HMSdrp. (A) Schematic description of oligonucleotide probes used to assess rRNA processing by Northern blot analysis and primer extension. Probes \#1-4 are partially or fully complementary to $16 \mathrm{~S}$ or $23 \mathrm{~S}$ rRNA precursor sequences (dashed lines) that are absent in mature rRNAs (thick lines). Probes \#5-6 anneal 15-35 and 35-55 nt downstream from the mature $16 \mathrm{~S}$ and $23 \mathrm{~S}$ rRNA $5^{\prime}$ ends, respectively. (B) Northern blot analysis of RNA samples isolated from HMS174(DE3) (lane 1) and HMSdrp (lane 2) with probes \#1-4 (four left panels). (Right panel) The agarose gel stained with ethidium bromide. The positions of 16S and 23S rRNAs are indicated. (C) Primer extension of total RNAs from HMS174(DE3) (lanes 1,2) and HMSdrp (lanes 3,4) using probes $\# 5$ and \#6, specific for $16 \mathrm{~S}$ and $23 \mathrm{~S}$ rRNA, respectively. (Lanes 2,4) Control lanes where no reverse transcriptase was added. (Lane L) A 10-bp ladder. The positions of the oligonucleotide probes $(\mathrm{O})$, and the reverse-transcription products derived from the mature (M) and precursor $(\mathrm{P})$ rRNAs are noted. The ratios of the amounts of precursor to mature rRNAs are indicated above the gels.

gene. A number of genes were found to be differentially regulated between HMSdrp and the other strains. In particular, the signals for the intergenic regions between the tandem quadruplicate argVYZQ tRNA genes, which 
map to $60.7 \mathrm{~min}$ of the E. coli chromosome and which encode $\mathrm{RNA}^{\mathrm{Arg} 2}$, were dramatically reduced or absent (Fig. $2 \mathrm{~A})$. The reduction suggested the possibility of a lesion in this region of the chromosome, a suspicion that was confirmed by PCR analysis (data not shown). Sequencing of this region revealed a 685 base pair (bp) deletion of the chromosome, which resulted in the loss of a cluster of three tRNA genes, as well as the corresponding intergenic regions (Fig. 2B). Hereafter, we refer to this deletion as $\triangle \arg Y Z Q$.

One expected consequence of the $\triangle \arg Y Z Q$ deletion is reduced expression of $\mathrm{tRNA}^{\mathrm{Arg} 2}$ in the cell. This was confirmed by Northern blot analysis, which indicated that the
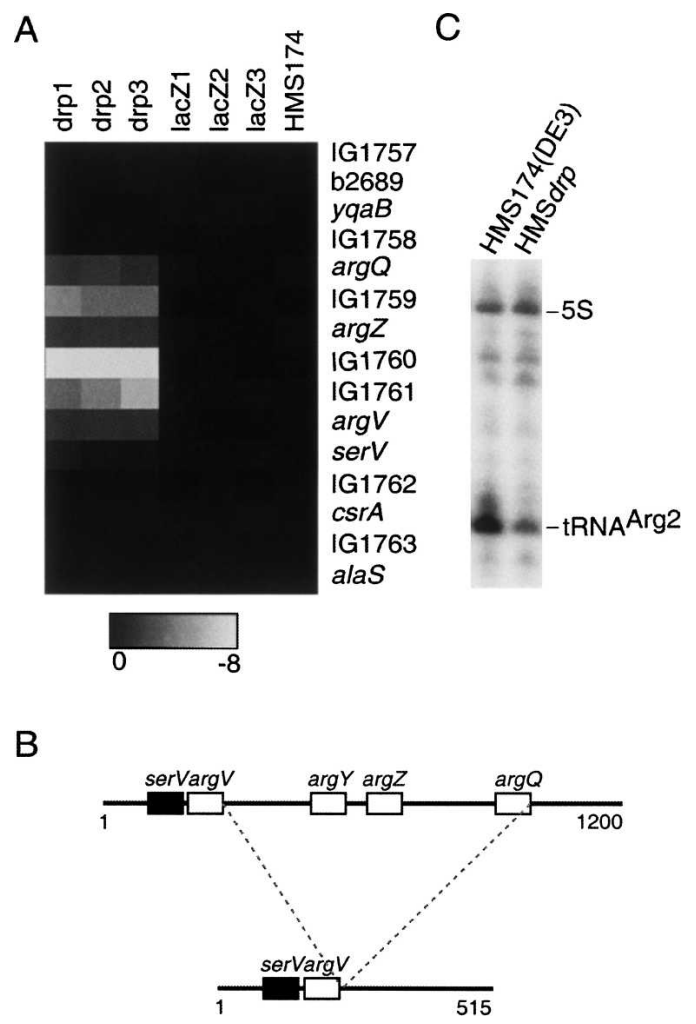

FIGURE 2. Microarray and Northern blot analysis. (A) Visualization of the microarray results corresponding to the serV/argV region. Three independent analyses of total RNA are shown from HMS $d r p$ (drp1, drp2, and drp3) and from a control strain containing a disruption in lacZ (lacZ1, lacZ2, and lacZ3), and one analysis of RNA from HMS174(DE3). The levels of downregulation of the genes and intergenic regions within and abutting the $\operatorname{serV} / \arg V$ region (IG1757 to alaS) are shown on a $\log _{2}$ scale using a gray-scale shading scheme: (black) no change in mRNA expression; (white) a 256-fold $\left(\log _{2}=-8\right)$ downregulation of gene expression. (B) Schematic of the $\operatorname{argVYZQ}$ operon. This operon comprises the serV tRNA gene followed by four identical genes ( $\operatorname{argV}, \arg Y, \arg Z$, and $\operatorname{argQ}$ ) encoding tRNA ${ }^{\mathrm{Arg} 2}$. A chromosomal deletion between $\arg V$ and $\operatorname{argQ}$, as depicted, results in the loss of three of these tRNA genes in HMSdrp. (C) Northern blot analysis. RNA samples derived from HMS and HMSdrp were analyzed using oligonucleotide probe \#7, complementary to tRNA ${ }^{\mathrm{Arg} 2}$. To correct for differences in RNA loading, the blot was also probed with an oligonucleotide complementary to $5 S$ rRNA (probe \#8). level of tRNA ${ }^{\mathrm{Arg} 2}$ in HMSdrp was approximately one-fourth of the level in the parental strain (Fig. 2C). This result is in agreement with the microarray analysis data, where tRNA ${ }^{\mathrm{Arg} 2}$ was found to be depressed approximately fivefold (Fig. 2A).

\section{Reduced tRNA ${ }^{\text {Arg2 }}$ levels result in rRNA processing defects}

The $\triangle \arg Y Z Q$ deletion in HMSdrp suggested that reduced expression of $\mathrm{tRNA}^{\mathrm{Arg} 2}$ could be responsible for the observed rRNA processing defects. To investigate this possibility, we transduced the $\triangle \arg Y Z Q$ deletion into the MG1655 strain background using a linked marker ( $z f h$ 3131::Tn10). We then compared rRNA processing defects in this strain (CJ1892) with an isogenic derivative (CJ1891=MG1655 $z f h-3131:: T n 10)$ that retains a wild-type $\operatorname{argVYZQ~locus.~Primer~extension~analysis~of~RNA~isolated~}$ from CJ1891 and CJ1892 indicated that the amount of unprocessed $23 \mathrm{~S}$ rRNA in the latter strain was more than threefold higher than in the former strain (Fig. 3A). This buildup indicated that rRNA processing defects were attributable to the $\triangle \arg Y Z Q$ deletion derived from HMSdrp, or to a mutation closely linked to this region of the chromosome. To directly evaluate whether reduced tRNA $^{\text {Arg2 }}$ levels were responsible for rRNA processing defects, we transformed strain HMSdrp with a plasmid containing the tRNA ${ }^{\mathrm{Arg} 2}$ gene (pArg2) under control of an arabinose-inducible promoter. Total RNA was isolated from cultures lacking or containing $0.2 \%$ arabinose, and rRNA was analyzed by primer extension using a $23 \mathrm{~S}$ rRNAspecific primer (probe \#6). As controls, HMS174(DE3) and HMSdrp were also transformed with a plasmid (pMPMT6) that lacks the tRNA ${ }^{\text {Arg2 }}$ gene. Expression of $\mathrm{tRNA}^{\mathrm{Arg} 2}$ from the plasmid was found to significantly reduce the levels of unprocessed $23 \mathrm{~S}$ rRNA, to levels close to those found in the wild-type strain (Fig. 3B, lanes 1,2,4). Thus, reduced $t R N A^{\mathrm{Arg} 2}$ dosage is primarily responsible for the rRNA processing defects seen in HMSdrp. Interestingly, CJ1892 grew slower than CJ1891 on LB-agar plates at $37^{\circ} \mathrm{C}$, similar to the growth defects that were observed in HMS $d r p$ relative to HMS174(DE3) (Fig. 3C), suggesting that reduced tRNA ${ }^{\text {Arg2 }}$ dosage also causes cell growth defects.

\section{Analysis of ribosomal fractions in strains underproducing tRNA ${ }^{\text {Arg2 }}$}

As rRNA processing and ribosome maturation are closely coupled, the possibility exists that defective rRNA processing observed in strains underproducing tRNA ${ }^{\text {Arg2 }}$ could indicate the accumulation of incomplete or defective ribosomes. For example, mutations in ribosomal proteins, or the lack of certain assembly factors, are associated with both the accumulation of rRNA precursors and defective ribosomal particles (Maguire and Wild 1997; Charollais et al. 2003, 2004). To investigate whether underproduction 
A

B

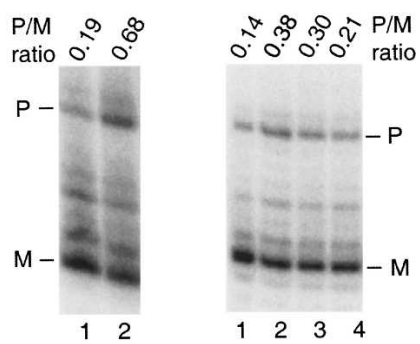

C
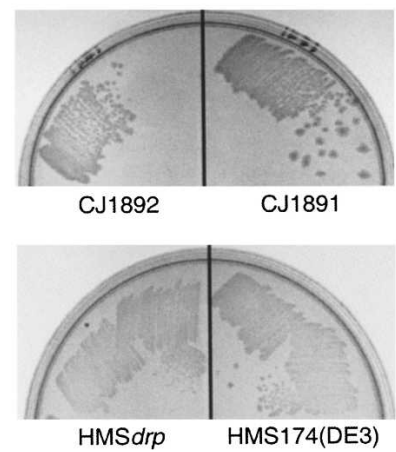

FIGURE 3. Growth and RNA analysis of strains containing one or four copies of the tRNA ${ }^{\text {Arg2 }}$ gene. (A) Primer extension analysis of RNAs derived from CJ1891 and CJ1892, using a 23S rRNA-specific probe. (Lane 1) CJ1891; (lane 2) CJ1892. The positions of mature (M) and precursor $(\mathrm{P})$ rRNAs are noted, as are the $\mathrm{P} / \mathrm{M}$ ratios. $(B)$ Complementation of the rRNA processing defects by expression of plasmid-encoded tRNA ${ }^{\text {Arg2 }}$. (Lane 1) HMS174(DE3)/pMPM-T6; (lane 2) HMSdrp/pMPM-T6; (lane 3) HMSdrp/pArg2-arabinose; (lane 4) HMSdrp/pArg2+arabinose. The corresponding $\mathrm{P} / \mathrm{M}$ ratios are noted. $(C)$ Growth phenotype of strains containing (CJ1892 and HMSdrp) or lacking [CJ1891 and HMS174(DE3)] the $\operatorname{argYZQ~}$ deletion, incubated at $37^{\circ} \mathrm{C}$ on LB-agar plates.

of tRNA ${ }^{\text {Arg2 }}$ causes such ribosomal particles to accumulate, we fractionated cell extracts derived from CJ1891 and CJ1892 by sucrose-gradient centrifugation, and generated ribosome profiles. A representative set of ribosome profiles is shown in Figure 4A. A comparison of these profiles indicated that there were no systematic differences between the profiles derived from the two strains.

Despite the inability to visualize defective ribosomal particles in CJ1892, it remained a possibility that incompletely assembled ribosomal particles, containing immature rRNA, were present at low levels. To investigate this possibility, we extracted RNA from fractions corresponding to $70 \mathrm{~S}$ ribosomes, to $50 \mathrm{~S}$ large ribosomal subunits, and to a fraction sedimenting at $\sim 40 \mathrm{~S}$, and then analyzed the RNA by primer extension using a $23 \mathrm{~S}$ rRNA-specific primer (Fig. $4 \mathrm{~B})$. The third fraction was chosen because mutations in several ribosome assembly factors are associated with the accumulation of pre-50S precursors that sediment at $40 \mathrm{~S}$ (Charollais et al. 2003, 2004). The primer extension analysis indicated that the $70 \mathrm{~S}$ and $50 \mathrm{~S}$ fractions derived from the two strains were equivalent, and contained the mature rRNA as well as $+3 / 4$ and $+7 / 8$ products (Fig. $4 B, C$ ). The lower proportion of immature rRNA in the 70S fraction is consistent with the previous, albeit yet unexplained, observation that final maturation of this rRNA occurs only when $50 \mathrm{~S}$ particles associate with $30 \mathrm{~S}$ subunits to form $70 \mathrm{~S}$ ribosomes (Srivastava and Schlessinger 1988). In contrast, when $40 \mathrm{~S}$ fractions were analyzed the levels of the $+7 / 8$ product were significantly higher in ribosomes derived
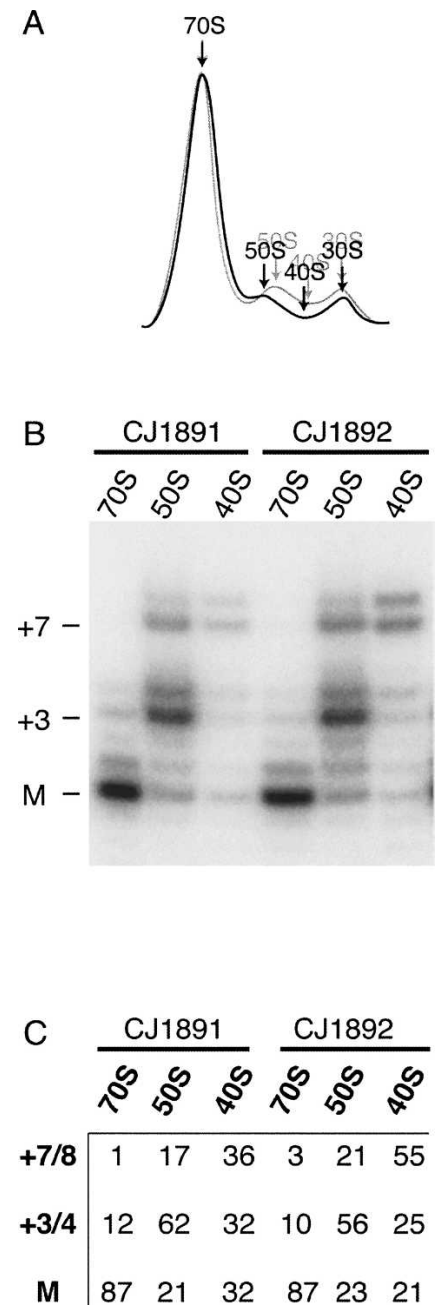

FIGURE 4. Ribosome profile analysis. (A) Superimposed images of the sucrose-gradient ultracentrifugation analysis of ribosome fractions derived from CJ1891 (black) and CJ1892 (gray). Each image is representative of three independent experiments. The positions of the $70 \mathrm{~S}$ ribosomes, $50 \mathrm{~S}$ large subunits, and $30 \mathrm{~S}$ small subunits are indicated by arrows. Also indicated is the position corresponding to $\sim 40$ S, from which fractions were isolated for primer extension analysis. (B) Primer extension analysis. RNA was isolated from 70S, $50 \mathrm{~S}$, and $40 \mathrm{~S}$ fractions, and analyzed by primer extension using probes specific for $23 \mathrm{~S}$ rRNA. The positions of the mature $(\mathrm{M}),+3$, and +7 products are indicated. $(C)$ Quantitation of mature and immature $23 \mathrm{~S}$ rRNA. The amounts of the mature, the $+3 / 4$, and the $+7 / 8$ products present in the fractions analyzed in $B$ were quantified using ImageQuant software, and are expressed as the percentage of the radioactivity present in all three products. 
from CJ1892 than in ribosomes derived from CJ1891. Whereas the mature, the $+3 / 4$, and the $+7 / 8$ products were present in roughly equal levels in CJ1891, the proportion of the $+7 / 8$ products was more than twice that of the mature or the $+3 / 4$ products in CJ1892. Thus, it appears that the $+7 / 8$ products accumulate to higher levels in $40 \mathrm{~S}$ fractions in CJ1892, than in the equivalent fractions in CJ1891. The sedimentation of the pre-23S rRNA at $\sim 40 \mathrm{~S}$ suggests that the RNA is associated in a complex containing an incomplete repertoire of ribosomal proteins.

\section{Additional defects in tRNA that result in accumulation of immature rRNAs}

The observation that reduced tRNA ${ }^{\mathrm{Arg} 2}$ levels affect rRNA processing suggested that other types of tRNA defects might have a similar effect. This possibility was tested in three different ways. First, rRNA processing was evaluated in a strain containing a temperature-sensitive mutation in RNase P. RNase P plays an essential role in tRNA processing, as it is required to generate mature tRNA $5^{\prime}$ ends (Altman et al. 1989). An isogenic pair of strains, one containing the temperature-sensitive rnpA49 mutation, was grown at $30^{\circ} \mathrm{C}$, and RNA was extracted after a shift to the nonpermissive temperature of $42^{\circ} \mathrm{C}$ (Kirsebom et al. 1988). By primer extension analysis, we found the mutant strain to contain substantially higher levels of unprocessed $16 \mathrm{~S}$ and $23 \mathrm{~S}$ rRNAs, than did the wild-type strain (Fig. 5A). Thus, the rRNA processing defects observed in RNase $\mathrm{P}$ mutant strains are likely to be a consequence of defective tRNA function. Alternatively, these defects could be explained by the fact that ribosomal operon RNA includes genes for tRNAs, and therefore defects in RNase P function can influence the processing of rRNAs. However, RNase P-mediated processing events are preceded by endonucleolytic cleavages that separate the pre-tRNA and prerRNA regions of the primary transcript (Srivastava and Schlessinger 1990); hence, it is unlikely that RNase P cleavages influence rRNA processing via a direct mechanism.

Second, a strain containing a single-base mutation (G7 to A7) at a position corresponding to the acceptor stem of the unique tRNA ${ }^{\text {Trp }}$ gene was examined. This mutation causes progressive denaturation and degradation of the tRNA as the temperature is increased (Eisenberg and Yarus 1980; Li et al. 2002). When RNA was extracted from this strain and from its wild-type counterpart after growth at $42^{\circ} \mathrm{C}$ and analyzed by primer extension, we found an increased level of unprocessed $16 \mathrm{~S}$ and $23 \mathrm{~S}$ rRNA in the mutant (Fig. 5B). Thus, tRNAs containing destabilizing mutations also cause rRNA processing defects.

Finally, we created a tRNA defect by reducing the availability of a tRNA by overexpression of an mRNA that contains multiple copies of codons for this tRNA. Strain CJ1825, which is unable to metabolize arabinose (Table 1), was transformed with plasmid pER133, which overexpresses

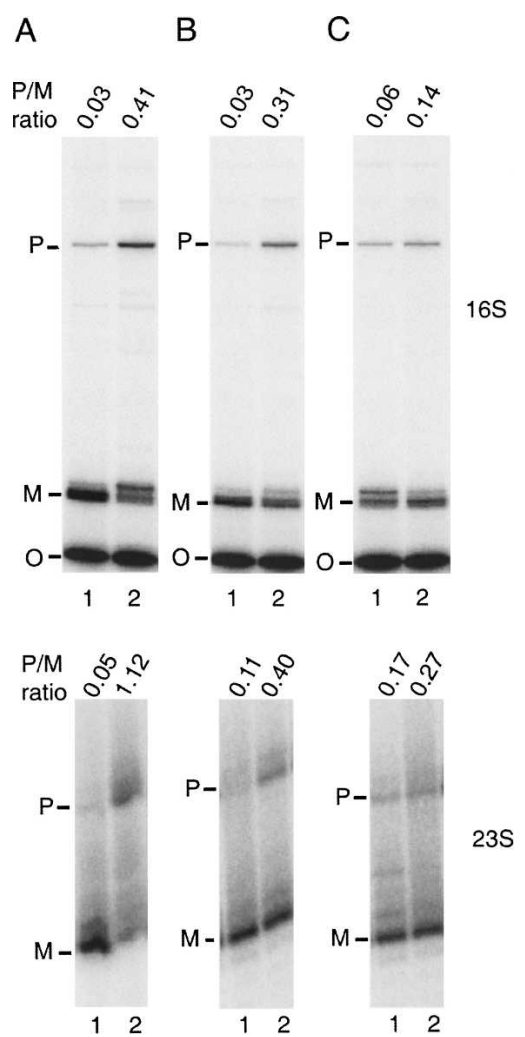

FIGURE 5. RNA analysis of strains containing additional tRNA defects. RNA was isolated from strains containing different tRNA defects and from isogenic wild-type strains, and rRNA processing was analyzed by primer extension with $16 \mathrm{~S}$ and $23 \mathrm{~S}$ rRNA-specific primers. Oligonucleotide primer $(\mathrm{O})$, precursor $(\mathrm{P})$, and mature $(\mathrm{M})$ products are noted, as are the $\mathrm{P} / \mathrm{M}$ ratios. $(A)$ Isogenic strains NHY312 and NHY322 (rnpA49) were grown at $30^{\circ} \mathrm{C}$ to midlog phase, followed by transfer to $42^{\circ} \mathrm{C}$ for $30 \mathrm{~min}$ prior to harvesting. RNAs were analyzed by primer extension using $16 \mathrm{~S}$ and $23 \mathrm{~S}$ rRNA-specific primers. (Lanes 1) RNA derived from NHY312; (lane 2) RNA derived from NHY322. (B) Strains CA244 and CA244tRNA ${ }^{\text {Trp(ts) }}$ were grown at $30^{\circ} \mathrm{C}$ to midlog phase, followed by transfer to $42^{\circ} \mathrm{C}$ for $30 \mathrm{~min}$ prior to harvesting. RNA was isolated from these strains and analyzed by primer extension. (Lane 1) CA244; (lane 2) CA244tRNA ${ }^{\operatorname{Trp}(\mathrm{ts})}$. (C) Strain CJ1825 was transformed with the control plasmid, pMPM-A4, or with pER133, a plasmid containing a short open reading frame that includes eight tandem AGA codons. The transformed strains were grown in $\mathrm{LB}+$ ampicillin at $37^{\circ} \mathrm{C}$, followed by the addition of arabinose to $0.2 \%$ when the strains were at midlog phase, to induce transcription of pER133. Cultures were harvested after further growth for 15 min, followed by RNA isolation and analysis. (Lanes 1,2) RNA isolated from CJ1825/pMPM-A4 and CJ1825/pER133 cultures, respectively.

an mRNA containing eight tandem AGA codons transcribed from an arabinose-regulated promoter. AGA codons are recognized by the minor tRNA species, $\mathrm{tRNA}^{\mathrm{Arg} 4}$, and arabinose induction of pER133-containing cells has been shown to reduce the availability of free $\mathrm{tRNA}^{\mathrm{Arg} 4}$, resulting in translation defects (Roche and Sauer 1999). Cultures of cells transformed with pER133 or a control plasmid were treated with arabinose for $15 \mathrm{~min}$, followed by RNA extraction and analysis by primer extension. This analysis revealed 
TABLE 1. Strains and plasmids used in this study

\begin{tabular}{|c|c|c|}
\hline A. Strain & Relevant genotype & Source or reference \\
\hline HMS174(DE3) & Wild-type & Perutka et al. 2004 \\
\hline HMSdrp & HMS174 (DE3) $\Delta \operatorname{argYZQ~rep::ItrB~}\left(T p^{R}\right)$ & Perutka et al. 2004 \\
\hline HMSlacZ & HMS174 (DE3) lacZ::ItrB & This study \\
\hline MG1655 & Wild-type & F. Blattner \\
\hline C)1891 & MG1655zfh-3131::Tn10 & This study \\
\hline C)1892 & CJ1891 $\arg Y Z Q$ & This study \\
\hline NHY312 & Wild-type & Kirsebom et al. 1988 \\
\hline NHY322 & NHY312rnpA49 & Kirsebom et al. 1988 \\
\hline CA244 & Wild-type & M.P. Deutscher \\
\hline $\mathrm{CA} 244^{\operatorname{Trp}(\mathrm{ts})}$ & CA244trpT(ts) & M.P. Deutscher \\
\hline C)1825 & $\Delta($ ara, leu $) 7697$ & Laboratory stock \\
\hline B. Plasmid & Relevant properties & Source or reference \\
\hline pMPM-T6 & $\begin{array}{l}\text { Cloning vector with pBAD promoter } \\
\text { sequences }\end{array}$ & Mayer 1995 \\
\hline pArg2 & pMPM-T6 derivative expressing tRNA ${ }^{\text {Arg2 }}$ & This study \\
\hline pMPM-A4 & $\begin{array}{l}\text { Cloning vector with pBAD promoter } \\
\text { sequences }\end{array}$ & Mayer 1995 \\
\hline pER133 & $\begin{array}{l}\text { pMPM-A4 derivative expressing an ORF } \\
\text { with eight AGA codons }\end{array}$ & Roche and Sauer 1999 \\
\hline
\end{tabular}

dramatic under rapid growth conditions, but are better tolerated under slow conditions of growth.

\section{DISCUSSION}

In this study, we demonstrate that a variety of defects in tRNAs can have a negative effect on rRNA maturation. Four types of tRNA defects were studied: a chromosomal deletion mutation that reduces tRNA abundance, a mutation in a tRNA processing enzyme (RNase P), a mutation that destabilizes a tRNA, and the titration of a tRNA that recognizes a rare codon. In each case, there was a significant increase in the amount of unprocessed rRNA. Thus, the consequences of defective tRNA function on rRNA processing are general. Further analysis of a strain that underproduces tRNA ${ }^{\text {Arg2 }}$ suggested that the unprocessed rRNA is assembled into that arabinose induction of an AGA-rich ORF enhanced $16 \mathrm{~S}$ and $23 \mathrm{~S}$ rRNA processing defects (Fig. 5C), indicating that tRNA availability also affects rRNA processing. Thus, each of the three additional functional tRNA defects examined had a negative effect on rRNA processing.

\section{Defects associated with underproducing tRNA ${ }^{\text {Arg2 }}$ are alleviated under slow growth conditions}

The production of ribosomes is known to be highly correlated with cell growth rates, whereas tRNA abundance changes to a lesser extent (Nomura et al. 1984; Dong et al. 1996). It is therefore possible that tRNAs are not limiting for translation under slow growth conditions, where they are present in relatively higher ratios over ribosomes, and cells may be able to tolerate tRNA defects better than under rapid growth conditions. To test this hypothesis, we compared the extent of rRNA processing between CJ1891 and CJ1892, under two conditions of slower growth: in minimal-glucose medium at $37^{\circ} \mathrm{C}$, and in $\mathrm{LB}$ medium at $16^{\circ} \mathrm{C}$. When $23 \mathrm{~S}$ rRNA processing was assessed, little difference in the amount of unprocessed rRNA between the wild-type and mutant strains was observed under either condition (Fig. 6A), in contrast to the significant differences observed in $\mathrm{LB}$ medium at $37^{\circ} \mathrm{C}$ (Fig. 3A). In addition, the differences in the colony sizes between CJ1891 and CJ1892 were significantly reduced on minimal-glucose plates at $37^{\circ} \mathrm{C}$, and on $\mathrm{LB}$ plates at $16^{\circ} \mathrm{C}$ (Fig. 6B), as compared to the much poorer growth of the mutant strain on LB-agar plates at $37^{\circ} \mathrm{C}$ (Fig. $3 \mathrm{~B}$ ). These observations suggest that tRNA-mediated defects are more incomplete ribosomal particles that sediment more slowly than the fully assembled particles.

The deletion that reduces RRNA $^{\operatorname{Arg} 2}$ abundance was identified in a strain that contains a disrupted rep gene. This deletion removes three of the four tRNA genes from the $\operatorname{argVYZQ}$ cluster, and presumably arises via homologous recombination between the first and fourth tRNA genes. By analogy with a similar deletion that suppresses a gyrB mutant, and because of DNA polymerase stalling in tRNA clusters, we propose both a mechanism and a selection for the $\triangle \arg Y Z Q$ deletion. The same deletion was identified in Salmonella typhimurium as a suppressor of a mutation in $g y r B$ encoding the second subunit of DNA gyrase (Blanc-Potard and Bossi 1994). DNA gyrase has a key role in DNA supercoiling, and mutations in this enzyme cause defects in a number of important cellular processes, including replication (Levine et al. 1998). Although the mechanism of gyrB suppression by this deletion mutation is not clear, it may be that mutations involving recombination between repeated DNA elements are favored in a gyrB genetic background in which DNA replication is aberrant. Likewise, a recombination event seems to have been facilitated or selected in a background lacking Rep. Rep has been characterized for its role in replication restart after replication fork collapse (Heller and Marians 2005), and recently the absence of Rep was shown to increase the frequency of short-homology-dependent illegitimate recombination within the E. coli chromosome as a result of replication arrest and DNA double-strand breaks (Shiraishi et al. 2005). In yeast, replication forks frequently slow down or stall at sites with multiple tRNA genes, and these genes are associated with DNA breaks 


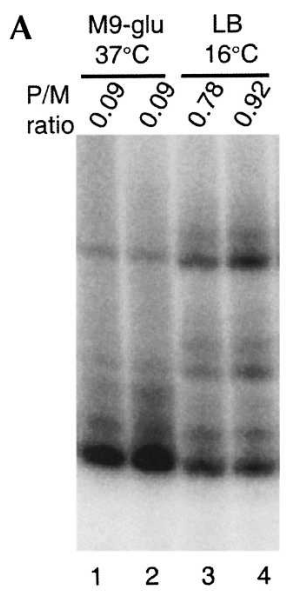

B

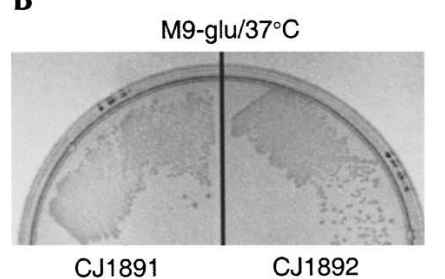

$\mathrm{LB} / 16^{\circ} \mathrm{C}$

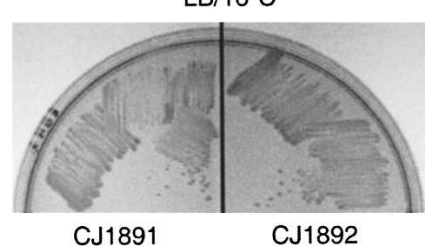

FIGURE 6. Processing of rRNA in strains grown under slow growth conditions. (A) Strains CJ1891 and CJ1892 were grown to midlog phase in minimal $\mathrm{M} 9$-glucose medium at $37^{\circ} \mathrm{C}$ or in $\mathrm{LB}$ medium at $16^{\circ} \mathrm{C}$, followed by RNA isolation and primer extension analysis of $23 \mathrm{~S}$ rRNA. The P/M ratio for each RNA sample is noted above the gel. (Lanes 1,3) CJ1891; (lanes 2,4) CJ1892. (B) Strains CJ1891 and CJ1892 were grown on a minimal M9-glucose plate at $37^{\circ} \mathrm{C}$ for $2 \mathrm{~d}$ or on an $\mathrm{LB}$ plate at $16^{\circ} \mathrm{C}$ for $1 \mathrm{wk}$.

(Deshpande and Newlon 1996; Ivessa et al. 2003) and chromosome instability (Admire et al. 2006). Both the presence of tRNA genes and directionality of DNA replication are suspected to cause instability, because tRNA genes transcribed into an oncoming replication fork stall the replication process (Deshpande and Newlon 1996). In line with these observations, it is feasible that the deletion of three tRNA ${ }^{\mathrm{Arg} 2}$ genes within the $\operatorname{argVYZQ}$ operon would relieve stalled forks resulting from the absence of Rep helicase, thereby giving cells a selective advantage. The resulting low expression of $\mathrm{tRNA}^{\mathrm{Arg} 2}$ would be tolerated by the cell because of the slow growth of the rep mutant.

Why is rRNA processing dependent on tRNA function? A likely possibility is that the tRNA-dependent translation elongation defects negatively impact the synthesis of cellular factors that are important for ribosome assembly. It has previously been shown that the addition of certain anti- biotics, such as chloramphenicol, also retards rRNA processing (Schlessinger et al. 1974). It is possible that the increased accumulation of rRNA precursors, due either to tRNA defects or to antibiotic treatment, has a common basis, which we speculate as the reduced production of one or more factors that have a role in ribosome biogenesis. In an analogous situation, a ts mutation in $\arg U$, the gene encoding the rare tRNA ${ }^{\mathrm{Arg} 4}$, was reported to cause DNA replication defects, probably because the mutant strain is unable to sufficiently produce one or more factors that play a role in replication (Henson et al. 1979; Garcia et al. 1986; Sakamoto et al. 2004).

Although the defects observed could be a result of general translation attenuation, thereby affecting the synthesis of a large number of proteins, three lines of evidence from data not shown suggest otherwise. First, when steadystate levels of proteins in HMS174(DE3) and HMSdrp were analyzed by polyacrylamide gel electrophoresis, no significant differences were observed, indicating the lack of a generalized translation defect. Second, when the time taken for appearance of $\beta$-galactosidase activity was measured after the addition of IPTG to cells containing one or four copies of the tRNA ${ }^{\text {Arg2 }}$ gene, no significant difference was observed between the two strains, suggesting a minimal influence of $\mathrm{tRNA}^{\mathrm{Arg} 2}$ availability on the translational elongation time for a reporter mRNA. Third, when the processing of several other classes of $E$. coli RNAs was analyzed in HMS174(DE3) and HMSdrp, using probes for 5S rRNA, as well as multiple tRNAs and small regulatory RNAs, no significant differences in precursor levels were observed, indicating a specific feedback on the $16 \mathrm{~S}$ and $23 \mathrm{~S}$ rRNAs. Furthermore, except for examples of extreme elongation defects, translation is mainly controlled at the level of initiation; with the exceptions generally involving mRNAs that exhibit programmed ribosome stalling or contain clusters of rare codons (Holm 1986; Chen and Inouye 1994; Kane 1995; Murakami et al. 2004; Mehra and Hatzimanikatis 2006).

In contrast, our data indicate that a reduction in the levels of the abundant $\mathrm{tRNA}^{\mathrm{Arg} 2}$ negatively influenced ribosome biogenesis, suggesting that certain biogenesisfactor-encoding mRNAs might instead be sensitive to small defects in translation elongation. Therefore, the processing defects observed for $16 \mathrm{~S}$ and $23 \mathrm{~S}$ rRNA appear to be relatively specific. In sum, the collective evidence supports a likelihood that the rRNA processing defects observed in this study are more likely due to reduced expression of one or a few factors that are specifically involved in ribosome biogenesis, and whose expression is particularly sensitive to defects in translation elongation.

What might these factors be? One possibility is that they are RNases directly involved in rRNA processing. Primer extension analysis indicated steps that follow the cleavage of ribosomal operon RNA by RNase III are retarded. The further maturation of these rRNAs requires several 
ribonucleases: $5^{\prime}$ maturation of $16 \mathrm{~S}$ rRNA is carried out by RNase E and RNase G, and 3' -end maturation of 23S rRNA is carried out by RNase $T$, whereas the enzymes required for $3^{\prime}$ maturation of $16 \mathrm{~S}$ rRNA or for $5^{\prime}$-end maturation of $23 \mathrm{~S}$ rRNA are not known (Deutscher 2006). It is possible that these enzymes are present in limiting quantities, so that their reduced expression could lead to an excess of substrate over product. Another possibility is that the ribosomal proteins themselves become limiting. It has been clearly established that accurate rRNA processing only occurs during the assembly of ribosomal proteins on the rRNA precursors (Srivastava and Schlessinger 1990). If the translation of ribosomal proteins were decreased, the assembly process could be disrupted or delayed, and rRNA precursors would accumulate. Other proteins that could be involved in this process could include ribosome assembly factors. Further studies will be needed to determine which factor(s) become limiting under conditions of tRNA defects. Independent of the mechanism, the studies described here demonstrate that defects in tRNAs affect translation not only directly, but also indirectly, by influencing ribosome biogenesis.

\section{MATERIALS AND METHODS}

\section{Materials}

Bulk chemicals and oligonucleotides were obtained from VWR or Sigma. Restriction enzymes, Polynucleotide kinase, and Taq DNA polymerase were obtained from New England Biolabs. Optikinase was purchased from US Biochemicals. Nylon membrane was obtained from Schleicher and Schuell. Radiochemicals were obtained from Perkin-Elmer.

\section{Strains and plasmids}

The relevant properties of strains and plasmids used in this study, and their sources are listed in Table 1 . New strains created for this study were made by $\mathrm{P} 1$ transduction or intron insertion (HMSlacZ) (Perutka et al. 2004). Plasmid pArg2 was made by PCR amplification of the $\operatorname{argV}$ gene using chromosomal DNA as a template and primers with the sequences $5^{\prime}$-AAAGGATCCCGAATCC CCGCCTCACCGCC-3' and 5'-TTTTTAAGCTTCACCTCTTCC ATACCTTCATCGC- $3^{\prime}$ as amplification primers. The PCR product was digested with BamHI and HindIII and ligated to plasmid pMPM-T6 digested with BglII and HindIII, resulting in pArg2.

\section{RNA analysis}

RNA was isolated from $5 \mathrm{~mL}$ cultures using either the RNeasy Kit (QIAGEN) or the hot phenol method as described previously (Jain et al. 2002). Primer extension analysis was carried out as previously described (Diwa et al. 2000), except that 5-10 fmol of labeled oligonucleotide and $0.3-1 \mu \mathrm{g}$ of RNA were used per reaction, and that annealing of labeled oligonucleotides to RNA was initiated by slow cooling to room temperature from $80^{\circ} \mathrm{C}$, instead of $65^{\circ} \mathrm{C}$. Oligonucleotides were labeled with $\gamma-{ }^{32} \mathrm{P}$ using Optikinase. The sequences of the probes used for analysis of rRNA processing are: probe \#1, 5' -TCGCTTAACCTCACAAC-3'; probe
\#2, 5'-GGCGTTGTAAGGTTAA-3'; probe \#3, 5'-AAAGTTTGA TGCTCAAAGAATTAAACTT-3'; and probe \#4, 5'-TCTTT AAGGTAAGGAGGTGA-3'. The sequences of the oligonucleotides used for primer extension analysis of $16 \mathrm{~S}$ and $23 \mathrm{~S}$ rRNA are probe \#5, 5'-CGTTCAATCTGAGCCATGATC-3' and probe \#6, 5'-CCTTCATCGCCTCTGACTGCC-3', respectively. The products of the primer extension reactions were separated on $8 \%$ or $20 \%$ acrylamide- $8 \mathrm{M}$ urea sequencing gels; the gels were dried and visualized by PhosphorImaging. Northern blot analysis was performed by fractionation of total RNA on a $0.7 \%$ agarose or a $6 \%$ acrylamide- $8 \mathrm{M}$ urea gel, transfered to a Nytran nylon membrane, and hybridization with oligonucleotide probes labeled with $\gamma-{ }^{32} \mathrm{P}$ using polynucleotide kinase. The probes used for quantifying tRNA ${ }^{\mathrm{Arg} 2}$ and $5 \mathrm{~S}$ rRNA abundance by Northern blot analysis are probe \#7, 5'-GAACCTCCGACCGCTCGGTTCG-3' and probe \#8, 5'-GGCGCTACGGCGTTTCACTTC-3'.

\section{Microarray analysis}

Samples from bacterial cultures, independently grown in LB medium at $30^{\circ} \mathrm{C}$ to an optical density of 0.5 at $600 \mathrm{~nm}$, were collected and immediately centrifuged for $1 \mathrm{~min}$ at 13,200 rpm. Pellets were snap-frozen in liquid nitrogen and stored at $-80^{\circ} \mathrm{C}$ until further treatment. Total RNA was isolated using an RNeasy Kit (QIAGEN). Processing and hybridization to Affymetrix GeneChip E. coli antisense genome arrays were performed according to the manufacturer's protocol. Three independent RNA preparations were used for HMSdrp and HMSlacZ, and one for HMS174(DE3). Data analysis was performed using GeneTraffic (IoBionLab) and parameters consistent with GC-RMA. An internal comparison among the HMSlacZ triplicates showed that 702 out of 7312 measured probes contained at least one value that deviated from the other two. These 702 probes were not used for further analysis. A comparison between HMSdrp and HMSlacZ showed that in the remaining data set there were 75 probe sets with a $P$ value of 0.01 or less, with $0.8 \%$ (58) of the probes repressed and $0.2 \%$ (17) induced.

\section{Ribosome analysis}

Ribosomes were analyzed by sucrose-gradient ultracentrifugation, as previously described (Gutgsell et al. 2005). Fractions of $1 \mathrm{~mL}$ were collected, and RNA was released from selected fractions by phenol extraction and ethanol precipitation, and analyzed by primer extension as described above.

\section{ACKNOWLEDGMENTS}

This work is dedicated to the memory of Dr. Robin Pietropaolo of the Wadsworth Center Microarray Core Facility. We thank Drs. Alan Lambowitz, Sidney Altman, Ken Rudd, Murray Deutscher, and Robert Sauer for providing strains or plasmids; Dr. Murray Deutscher for advice throughout the course of this work; and Dr. Andrew A. Reilly for assistance with microarray data analysis. This work was supported by start-up funds from the Lucille P. Markey Foundation to C.J., NIH grants GM39422 and GM44844 to M.B., and funding by the Swedish Research Council to J.G.S.-J.

Received September 28, 2006; accepted January 2, 2007. 


\section{REFERENCES}

Admire, A., Shanks, L., Danzl, N., Wang, M., Weier, U., Stevens, W., Hunt, E., and Weinert, T. 2006. Cycles of chromosome instability are associated with a fragile site and are increased by defects in DNA replication and checkpoint controls in yeast. Genes \& Dev. 20: $159-173$.

Altman, S., Baer, M.F., Bartkiewicz, M., Gold, H., Guerrier-Takada, C., Kirsebom, L.A., Lumelsky, N., and Peck, K. 1989. Catalysis by the RNA subunit of RNase P-A minireview. Gene 82: 63-64.

Blanc-Potard, A.B. and Bossi, L. 1994. Phenotypic suppression of DNA gyrase deficiencies by a deletion lowering the gene dosage of a major tRNA in Salmonella typhimurium. J. Bacteriol. 176: 22162226.

Charollais, J., Pflieger, D., Vinh, J., Dreyfus, M., and Iost, I. 2003. The DEAD-box RNA helicase SrmB is involved in the assembly of 50S ribosomal subunits in Escherichia coli. Mol. Microbiol. 48: 1253-1265.

Charollais, J., Dreyfus, M., and Iost, I. 2004. CsdA, a cold-shock RNA helicase from Escherichia coli, is involved in the biogenesis of $50 \mathrm{~S}$ ribosomal subunit. Nucleic Acids Res. 32: 2751-2759.

Chen, G.T. and Inouye, M. 1994. Role of the AGA/AGG codons, the rarest codons in global gene expression in Escherichia coli. Genes \& Dev. 8: 2641-2652.

Deshpande, A.M. and Newlon, C.S. 1996. DNA replication fork pause sites dependent on transcription. Science 272: 1030-1033.

Deutscher, M.P. 2006. Degradation of RNA in bacteria: Comparison of mRNA and stable RNA. Nucleic Acids Res. 34: 659-666.

Diwa, A., Bricker, A.L., Jain, C., and Belasco, J.G. 2000. An evolutionarily conserved RNA stem-loop functions as a sensor that directs feedback regulation of RNase E gene expression. Genes \& Dev. 14: 1249-1260.

Dong, H., Nilsson, L., and Kurland, C.G. 1996. Co-variation of tRNA abundance and codon usage in Escherichia coli at different growth rates. J. Mol. Biol. 260: 649-663.

Eisenberg, S.P. and Yarus, M. 1980. The structure and aminoacylation of a temperature-sensitive tRNATrp (Escherichia coli). J. Biol. Chem. 255: 1128-1137.

Garcia, G.M., Mar, P.K., Mullin, D.A., Walker, J.R., and Prather, N.E. 1986. The E. coli dnaY gene encodes an arginine transfer RNA. Cell 45: 453-459.

Gutgsell, N.S., Deutscher, M.P., and Ofengand, J. 2005. The pseudouridine synthase RluD is required for normal ribosome assembly and function in Escherichia coli. RNA 11: 1141-1152.

Heller, R.C. and Marians, K.J. 2005. Unwinding of the nascent lagging strand by Rep and PriA enables the direct restart of stalled replication forks. J. Biol. Chem. 280: 34143-34151.

Henson, J.M., Chu, H., Irwin, C.A., and Walker, J.R. 1979. Isolation and characterization of $d n a X$ and dnaY temperature-sensitive mutants of Escherichia coli. Genetics 92: 1041-1059.

Holm, L. 1986. Codon usage and gene expression. Nucleic Acids Res. 14: 3075-3087.

Ivessa, A.S., Lenzmeier, B.A., Bessler, J.B., Goudsouzian, L.K., Schnakenberg, S.L., and Zakian, V.A. 2003. The Saccharomyces cerevisiae helicase Rrm3p facilitates replication past nonhistone protein-DNA complexes. Mol. Cell 12: 1525-1536.

Jain, C., Deana, A., and Belasco, J.G. 2002. Consequences of RNase E scarcity in Escherichia coli. Mol. Microbiol. 43: 1053-1064.

Kane, J.F. 1995. Effects of rare codon clusters on high-level expression of heterologous proteins in Escherichia coli. Curr. Opin. Biotechnol. 6: 494-500.
King, T.C., Sirdeshmukh, R., and Schlessinger, D. 1984. RNase III cleavage is obligate for maturation but not for function of Escherichia coli pre-23S rRNA. Proc. Natl. Acad. Sci. 81: 185-188.

King, T.C., Sirdeskmukh, R., and Schlessinger, D. 1986. Nucleolytic processing of ribonucleic acid transcripts in procaryotes. Microbiol. Rev. 50: 428-451.

Kirsebom, L.A., Baer, M.F., and Altman, S. 1988. Differential effects of mutations in the protein and RNA moieties of RNase P on the efficiency of suppression by various tRNA suppressors. J. Mol. Biol. 204: $879-888$.

Levine, C., Hiasa, H., and Marians, K.J. 1998. DNA gyrase and topoisomerase IV: Biochemical activities, physiological roles during chromosome replication, and drug sensitivities. Biochim. Biophys. Acta 1400: 29-43.

Li, Z., Reimers, S., Pandit, S., and Deutscher, M.P. 2002. RNA quality control: Degradation of defective transfer RNA. EMBO J. 21: $1132-1138$.

Maguire, B.A. and Wild, D.G. 1997. The effects of mutations in the rpmB,G operon of Escherichia coli on ribosome assembly and ribosomal protein synthesis. Biochim. Biophys. Acta 1353: 137-147.

Mayer, M.P. 1995. A new set of useful cloning and expression vectors derived from pBlueScript. Gene 163: 41-46.

Mehra, A. and Hatzimanikatis, V. 2006. An algorithmic framework for genome-wide modeling and analysis of translation networks. Biophys. J. 90: 1136-1146.

Murakami, A., Nakatogawa, H., and Ito, K. 2004. Translation arrest of SecM is essential for the basal and regulated expression of SecA. Proc. Natl. Acad. Sci. 101: 12330-12335.

Nomura, M., Gourse, R., and Baughman, G. 1984. Regulation of the synthesis of ribosomes and ribosomal components. Annu. Rev. Biochem. 53: 75-117.

Perutka, J., Wang, W., Goerlitz, D., and Lambowitz, A.M. 2004. Use of computer-designed group II introns to disrupt Escherichia coli DExH/D-box protein and DNA helicase genes. J. Mol. Biol. 336: 421-439.

Rocak, S. and Linder, P. 2004. DEAD-box proteins: The driving forces behind RNA metabolism. Nat. Rev. Mol. Cell Biol. 5: 232-241.

Roche, E.D. and Sauer, R.T. 1999. SsrA-mediated peptide tagging caused by rare codons and tRNA scarcity. EMBO J. 18: 4579-4589.

Sakamoto, K., Ishimaru, S., Kobayashi, T., Walker, J.R., and Yokoyama, S. 2004. The Escherichia coli $\operatorname{argU10}$ (Ts) phenotype is caused by a reduction in the cellular level of the $\arg U$ tRNA for the rare codons AGA and AGG. J. Bacteriol. 186: 5899-5905.

Schlessinger, D., Ono, M., Nikolaev, N., and Silengo, L. 1974. Accumulation of $30 \mathrm{~S}$ preribosomal ribonucleic acid in an Escherichia coli mutant treated with chloramphenicol. Biochemistry 13: $4268-4271$.

Shiraishi, K., Imai, Y., Yoshizaki, S., and Ikeda, H. 2005. Rep helicase suppresses short-homology-dependent illegitimate recombination in Escherichia coli. Genes Cells 10: 1015-1023.

Srivastava, A.K. and Schlessinger, D. 1988. Coregulation of processing and translation: Mature 5' termini of Escherichia coli $23 \mathrm{~S}$ ribosomal RNA form in polysomes. Proc. Natl. Acad. Sci. 85: 7144-7148.

Srivastava, A.K. and Schlessinger, D. 1990. Mechanism and regulation of bacterial ribosomal RNA processing. Annu. Rev. Microbiol. 44: 105-129.

Tanner, N.K. and Linder, P. 2001. DExD/H box RNA helicases: From generic motors to specific dissociation functions. Mol. Cell 8: 251-262.

Williamson, J.R. 2003. After the ribosome structures: How are the subunits assembled? RNA 9: 165-167. 

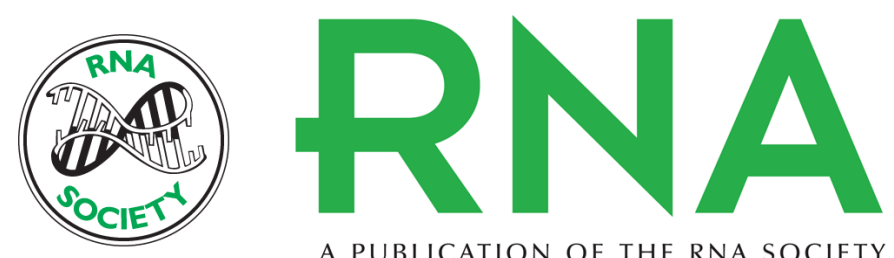

A PUBLICATION OF THE RNA SOCIETY

\section{Functional defects in transfer RNAs lead to the accumulation of ribosomal RNA precursors}

Jacoba G. Slagter-Jäger, Leopold Puzis, Nancy S. Gutgsell, et al.

RNA 2007 13: 597-605 originally published online February 9, 2007

Access the most recent version at doi:10.1261/rna.319407

References This article cites 39 articles, 17 of which can be accessed free at:

http://rnajournal.cshlp.org/content/13/4/597.full.html\#ref-list-1

License

Email Alerting Receive free email alerts when new articles cite this article - sign up in the box at the

Service top right corner of the article or click here.

To subscribe to $R N A$ go to:

http://rnajournal.cshlp.org/subscriptions 